BMJ Open

Respiratory

Research

\title{
Mindfulness-based stress reduction in patients with interstitial lung diseases: a pilot, single-centre observational study on safety and efficacy
}

\author{
Giacomo Sgalla, ${ }^{1}$ Stefania Cerri, ${ }^{2}$ Roberto Ferrari, ${ }^{3}$ Maria Pia Ricchieri, ${ }^{4}$ \\ Stefano Poletti, ${ }^{5}$ Margherita Ori, ${ }^{2}$ Martina Garuti, ${ }^{2}$ Gloria Montanari, ${ }^{2}$ \\ Fabrizio Luppi, ${ }^{2}$ Kyriakoula Petropulacos, ${ }^{6}$ Luca Richeldi ${ }^{1}$
}

To cite: Sgalla G, Cerri S, Ferrari R, et al. Mindfulnessbased stress reduction in patients with interstitial lung diseases: a pilot, singlecentre observational study on safety and efficacy. BMJ Open Resp Res 2015;2: e000065. doi:10.1136/ bmjresp-2014-000065

Received 18 September 2014 Revised 11 December 2014 Accepted 12 December 2014

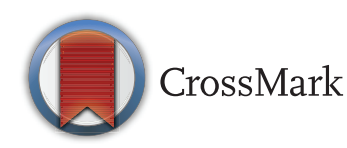

For numbered affiliations see end of article.

Correspondence to Dr Giacomo Sgalla; giacomo.sgalla@gmail.com

\section{ABSTRACT}

Background: Chronic, progressive respiratory symptoms are associated with great psychological and emotional impact in patients suffering from interstitial lung disease (ILD). This single-centre pilot study evaluated for the first time the safety, feasibility and efficacy of a Mindfulness Based Stress Reduction Program (MBSR) in a group of patients with ILD.

Methods: Prospective observational study set in a university hospital ILD outpatient clinic. Nineteen patients with different ILDs were recruited 2 months prior to the start of the 8-week MBSR program and followed up for 12 months. Primary outcomes were program safety and feasibility, while secondary outcomes were changes in moods and stress (assessed by Profile Of Mood State (POMS) and Perceived Stress Scale (PSS) questionnaires), symptoms (Shortness Of Breath (SOB) and Cough And Sputum Assessment (CASA-Q) questionnaires), lung function and exercise tolerance at 12 months.

Results: Two patients (10.5\%) dropped out in the observational period before the start of the MBSR intervention because of non-respiratory causes. All 17 patients who entered the 8-week MBSR program managed to complete it with an adherence average of eight sessions of nine. No adverse events related to the mindfulness training were reported. Statistically significant improvements in the POMS total score and in several individual items of POMS and PSS were observed throughout the study. However, respiratory questionnaire scores, lung function and exercise tolerance did not show a significant difference over time.

Conclusions: An MBSR program appears to be safe and feasible in patients with ILD, and might affect perceived moods and stress producing a positive and lasting improvement in several stress-related negative domains. These findings pave the way to larger (possibly multicentre), randomised, controlled confirmatory trials.

\section{INTRODUCTION}

Interstitial lung diseases (ILDs) are a large and heterogeneous group of conditions characterised by the diffuse involvement of the

\section{KEY MESSAGES}

Interstitial Lung Diseases (ILDs) are characterised by symptoms such as chronic, dyspnea and cough. These lead to significant psychological distress, which is scarcely manageable.

- This study represents the first attempt to conduct a Mindfulness-Based Stress Reduction (MBSR) program in a group of patients with ILD.

MBSR appears to be a safe and feasible approach in patients with ILD at different stages of severity, and it might produce significant improvement of mood and perception of stress.

pulmonary interstitial space, with different patterns of inflammation and fibrosis. Many ILDs are rare entities if considered separately, although together they account for one-third of the overall respiratory morbidity. Chronic ILDs may result in the onset of pulmonary fibrosis, consisting in the aberrant deposition of connective tissue (scar) in the interstitium, possibly leading to impairment of gas exchange and ultimately death from respiratory failure. Idiopathic pulmonary fibrosis (IPF), one of the most common ILDs, is a specific form of chronic, progressive interstitial pneumonia of unknown cause, associated with a poor prognosis with an average duration from diagnosis to time of death of 2-3 years. ${ }^{12}$

Chronic, progressive shortness of breath is nearly universal in patients with fibrotic ILDs, and may be accompanied by other invalidating symptoms such as fatigue and cough. These affect the quality of life of these individuals by limiting daily life activities, with consequences on the psychological and emotional levels. The anxiety related to fear of such symptoms and consequent loss of self-confidence can 
generate further disability, thus triggering a dangerous vicious circle. Basal dyspnoea is negatively correlated with quality of life and survival in IPF, ${ }^{3}$ and longitudinal changes in dyspnoea also predict prognosis. ${ }^{5}$ However, despite the clinical significance of psychological and emotional disturbance in ILD, there is paucity of data about its appropriate management; disease-centred pharmacological therapies have shown little benefit, and complementary management strategies are frequently needed, including pulmonary rehabilitation, supplemental oxygen and opioids. ${ }^{6}$ Available interventions that could have an impact on psychological aspects of the disease may include individual psychological counselling, participation inpatient support groups or attendance at mindfulness programs: however, to the best of our knowledge, the effect of these interventions has never been objectively measured.

The term 'mindfulness' refers to a conscious modality characterised by a state of mental presence and attention to the present moment. ${ }^{7}$ Mindfulness-based techniques teach the capacity of discriminating thoughts, emotions and actions with positive effects from those with harmful effects, increasing the awareness that thoughts are not reality but only one of its representations.

Mindfulness-Based Stress Reduction (MBSR) is a standardised mindfulness training program created in 1979 by $\mathrm{J}$ Kabat-Zinn to integrate the ancient practices of meditation with contemporary clinical and psychological practice. ${ }^{8}$ Initially, it was developed in an experimental way to treat groups of patients suffering from chronic pain, ${ }^{9}$ but after its standardisation it has been largely adopted for treating other disorders in the medical and psychological fields. In cancer, mindfulness groups were shown to develop acceptance to the progression of diseases, reduction of fear and avoidance drives. ${ }^{10}$ Critical reviews of the evidence of the effect of mindfulness intervention in this field are encouraging, ${ }^{11}{ }^{12}$ with clinically relevant implications to alleviate psychological and physical suffering of persons living with cancer.

MBSR has also demonstrated efficacy in reducing negative moods and the perceived stress related to dyspnoea in asthmatic patients in a pilot study. ${ }^{13}$ MBSR programs show efficacy in improving the health-related quality of life, ${ }^{14}$ confirming the reliability of these studies and suggesting long-term follow-up and the use of active control groups.

Fibrotic lung diseases, often characterised by the impairment of quality of life due to progressive symptoms and worsening emotional status, ${ }^{15} 16$ may represent another potential application field for MBSR, but has not currently been investigated.

In this study, we sought to assess the safety and the feasibility of an MBSR program in a group of patients affected by different ILDs. We investigated the efficacy of MBSR on mood, quality of life and also pulmonary function, as we hypothesised that mindfulness practice might indirectly trigger a positive, measurable effect on pulmonary performance and exercise tolerance.
METHODS

\section{Study population and design}

Nineteen adult patients were recruited between November and December 2012 at the Centre for Rare Lung Diseases of the University Hospital of Modena, Italy. Inclusion criteria included a confirmed diagnosis of ILD according to international guidelines. Exclusion criteria were the inability to express a valid consent, the inability to perform pulmonary function tests, the use of long-term oxygen therapy for more than $18 \mathrm{~h} /$ day and the regular practice of meditation or Yoga, as the study was meant to evaluate the efficacy of mindfulness-based practice including meditation and Hatha Yoga techniques introduced for the first time. Patients of all levels of disease were included, as the purpose of the study was to evaluate for the first time the feasibility of an MBSR program in a heterogeneous sample of patients with ILD. We conducted a prospective, observational study with a duration of 1 year, comprising three phases: a preintervention observational period ( 2 months), an intervention period ( 2 months) during which patients were trained in the MBSR program, and a postintervention observational period (8 months; figure 1).

\section{Ethics and recruitment}

The conduct of the study was approved by the Ethics Committee of Modena, Italy. Twenty-five patients followed up at the Centre for Rare Lung Diseases of the University Hospital of Modena were invited to take part and provided with a participant information sheet. An orientation meeting was also held in order to introduce candidates to the basic concepts of mindfulness and give information about the MBSR program. Written informed consent was collected from all patients who decided to participate. Overall, six patients declined participation, mainly due to the practical difficulties they would have encountered in attending the MBSR program (many patients lived far away from the centre).

\section{MSBR program}

The program was conducted by an MBSR instructor with a personal history of 20 years of supervised meditation practice and who was specifically trained for 2 years in mindfulness methods at the University of Florence (Italy). It was divided into eight weekly meetings of $150 \mathrm{~min}$ each, to which all the patients were invited to join as a single group. Meetings were held in a comfortable quiet room located inside the hospital building. Three different techniques were taught in sequence: body scan focusing on sensations from different parts of the body, with verbal reminders from the instructor to focus on abdominal breathing; sitting meditation on a chair with a straight back, bringing not judgemental awareness of natural breath and on mental events; light yoga based on instruction in simple body movements and postures with controlled breathing. Instructions were given to patients to continue performing exercises daily at home for $45 \mathrm{~min}$ between the weekly meetings and after the 


\begin{tabular}{|c|c|c|c|c|}
\hline $\begin{array}{l}\text { November-December } \\
2012\end{array}$ & January-March 2013 & $\begin{array}{l}\text { June } \\
2013\end{array}$ & $\begin{array}{l}\text { Sept. } \\
2013\end{array}$ & $\begin{array}{l}\text { Nov. } \\
2013\end{array}$ \\
\hline
\end{tabular}

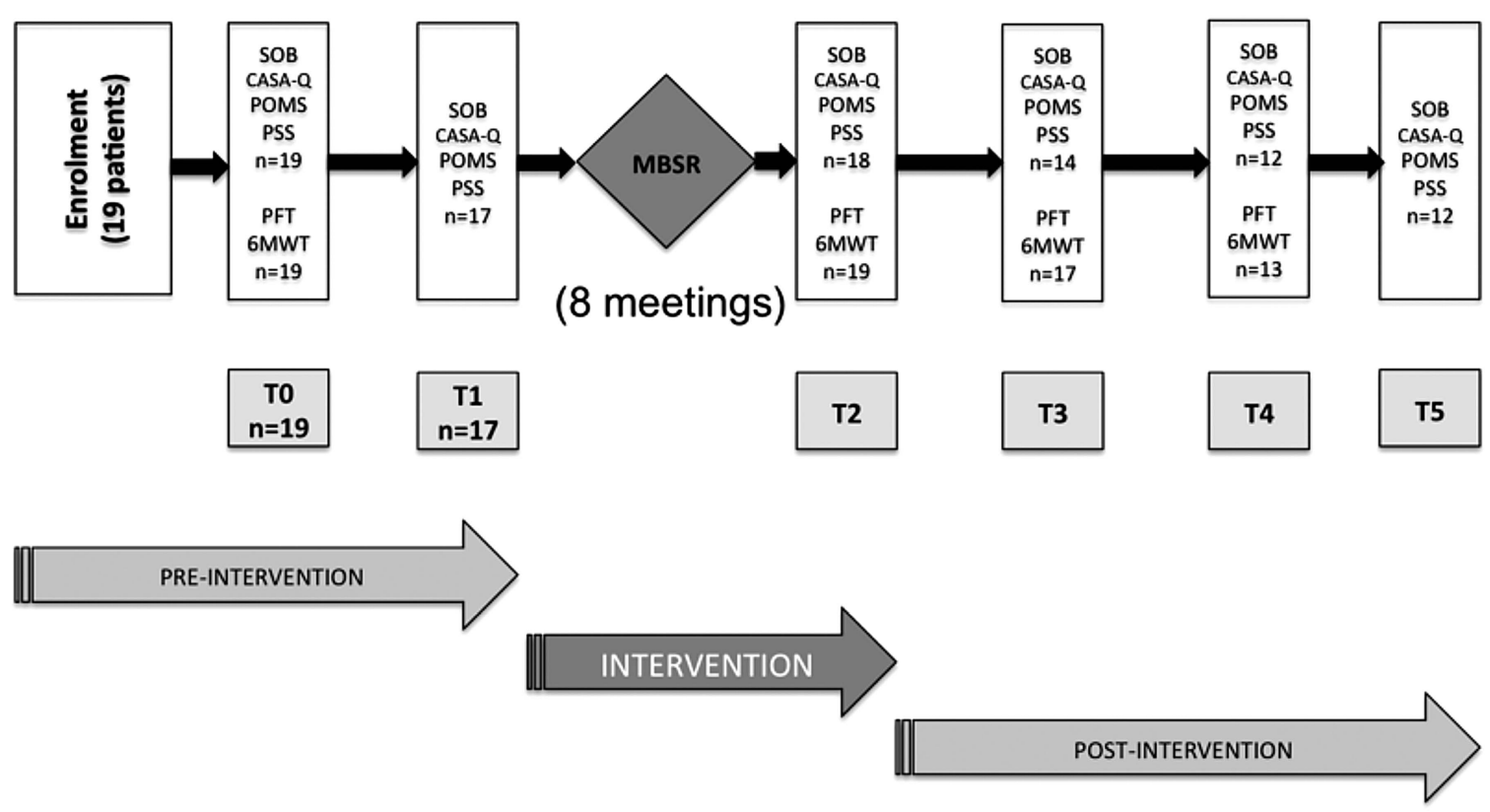

Figure 1 Study design.

SOB, Shortness Of Breath questionnaire; CASA-Q, Cough And Sputum Assessment Questionnaire; POMS, Profile Of Mood State test; PSS, Perceived Stress Scale; PFT, pulmonary function test; 6MWT, 6 min walk test; MBSR, Mindfulness Based Stress Reduction program.

training programme was completed; patients were also provided with CDs as a guide for home practice. An all-day meeting of $7 \mathrm{~h}$ between the sixth and the seventh session completed the course. Overall, the instructor total contact time was $28 \mathrm{~h}$ in 8 weeks.

At the end of the program, patients were asked to continue practising alone at home every day. To support patients' home practice, three quarterly group meetings of $150 \mathrm{~min}$ each were added during the postintervention observational period.

\section{Outcome measures}

The primary outcomes of the study were safety, measured as the frequency of adverse events related to the practice of mindfulness techniques, and feasibility, measured as the rate of dropouts and adherence to the MBSR program. Other outcomes included changes in mood, perceived stress and respiratory symptoms (measured by patient-reported outcomes-questionnaires), change in lung function (assessed by the rate of relative change from baseline in forced vital capacity (FVC, \% predicted), and rate of change in Single Breath-Diffusion Lung Capacity of Carbon Oxide (SB-DLCO) and change in exercise tolerance measured as changes in the $6 \mathrm{~min}$ walking test $(6 \mathrm{MWT})$.

The Profile Of Mood State (POMS) test, ${ }^{17}$ measuring the improvement of mood, consists of 40 items clustered into 6 scales (anger, confusion, depression, fatigue, tension, Vigour). The Perceived Stress Scale (PSS) ${ }^{18}$ measures the reduction of stress and consists of 10 items corresponding to 10 different domains (anger, perception of control, irritability, self-trust, expectations, efficacy, control of irritation, self-control, uncontrollable anger, excessive difficulties).

The University of California San Diego Shortness Of Breath Questionnaire (UCSD-SOB) ${ }^{19}$ and Cough And Sputum Assessment Questionnaire (CASA-Q), ${ }^{20}$ respectively assess self-reported shortness of breath and impact of cough while performing a variety of activities of daily living.

Questionnaires were administered at: enrolment (T0), at the beginning and at the end of the intervention ( $\mathrm{T} 1$ and T2), and then at each quarterly meeting until the end of the follow-up (T3, T4 and T5).

\section{Statistical analysis}

For POMS and PSS tests, average scores and changes in scores were evaluated at the beginning and the end of the study and also between the different intervals of the follow-up. For SOB and CASA-Q, total scores for each patient were calculated and then changes in the scores were evaluated for statistical significance between the beginning and the end of the study.

Lung function and exercise tolerance were also measured for each patient approximately every 3 months. 
Spirometry and SB-DLCO were performed according to the available international guidelines, ${ }^{21-23}$ as well as the 6MWT (distance covered) ${ }^{24}$ The Wilcoxon test was used to calculate the statistical significance of the changes in the average scores of the POMS and PSS questionnaires between the different times of the follow-up.

A non-parametric test for repeated measures was used to assess changes in lung function tests (FVC and SB-DLCO), 6MWD and questionnaire scores for SOB and CASA-Q between T0, T2 and T4. To handle missing answers in items of the SOB and CASA-Q we chose to calculate the total score relatively to the maximum theoretical score based on the number of answers provided.

\section{RESULTS}

\section{Baseline characteristics}

The main baseline characteristics are shown in table 1 . Eleven men and eight women (mean age \pm SD: 65 \pm 8 years) formed a single MBSR training group. The majority of participants (12 of 19) had a diagnosis of IPF. The remaining seven patients represented a broad spectrum of ILD.

\section{Safety and feasibility}

Two patients (10.5\%) dropped out early from the study for non-respiratory causes (low back pain and chronic

Table 1 Demographic and clinical features of the population

\begin{tabular}{|c|c|}
\hline & $\begin{array}{l}\text { Patients enrolled } \\
(\mathrm{N}=19)\end{array}$ \\
\hline Age, years (mean $\pm S D)$ & $65 \pm 8$ \\
\hline$M / F, n$ & $11 / 8$ \\
\hline IPF, n & 12 \\
\hline Other ILDs, n & 7 \\
\hline NSIP & 2 \\
\hline RA-ILD & 1 \\
\hline Chronic-HP & 1 \\
\hline CTD-ILD & 1 \\
\hline Asbestosis & 1 \\
\hline DIPNECH & 1 \\
\hline \multicolumn{2}{|l|}{ Lung function tests at baseline } \\
\hline $\begin{array}{l}\text { Per cent of predicted FVC } \\
\text { (mean } \pm \text { SD) }\end{array}$ & $82 \pm 26$ \\
\hline $\begin{array}{l}\text { Per cent of predicted SB-DLCO } \\
(\text { mean } \pm S D)\end{array}$ & $51 \pm 19$ \\
\hline $\begin{array}{l}\text { 6MWD at baseline, metres } \\
(\text { mean } \pm S D)\end{array}$ & $499 \pm 87$ \\
\hline Dropouts, n (\%) & $2(10.5 \%)$ \\
\hline $\begin{array}{l}\text { Average number of sessions } \\
\text { attended (of } 9 \text { ) }\end{array}$ & 8 \\
\hline \multicolumn{2}{|c|}{$\begin{array}{l}\text { 6MWD, 6-min walking test distance; CTD-ILD, connective tissue } \\
\text { disease associated interstitial lung disease; DIPNECH, diffuse } \\
\text { idiopathic pulmonary neuroendocrine cell hyperplasia; FVC, forced } \\
\text { vital capacity; HP, hypersensitivity pneumonitis; ILDs, interstitial } \\
\text { lung diseases; IPF, idiopathic pulmonary fibrosis; NSIP, } \\
\text { non-specific interstitial pneumonia; RA-ILD, rheumatoid arthritis } \\
\text { associated interstitial lung disease; SB-DLCO, single breath } \\
\text { diffusion lung capacity for CO. }\end{array}$} \\
\hline
\end{tabular}

fatigue, respectively) before they had started the intervention, and were not included in the analysis. The remaining 17 patients completed the 8-week intervention period. One patient suffered from acute deterioration of clinical conditions during the intervention period and was admitted into the ward of the clinic of respiratory diseases, but still managed to attend the meetings during the period of hospitalisation. There were no adverse events related to the activities performed, either during the intervention period or during the follow-up, when patients continued the daily home practice. The average number of sessions attended per patient was eight of nine. Adherence to homework was not recorded.

\section{POMS and PSS}

The results for the average scores of POMS and PSS tests are reported in figure 2. The POMS test showed significant changes in the mean scores indicating an improvement of mood throughout the study period. A total score was calculated by the addition of all the variables except for vigour, whose scores were subtracted. A statistically significant $(\mathrm{z}=2.197, \mathrm{p}=0.028)$ reduction can be found between the start and the end of the study. This reduction becomes more evident after the intervention (from T2 on), while a slight trend towards a worsening of mood was seen during the intervention period. Between $\mathrm{T} 0$ and $\mathrm{T} 5$, that is, between enrolment and the end of the study, the scores for three scales showed a statistically significant reduction: fatigue $(\mathrm{z}=2.362$, $\mathrm{p}=0.018)$, depression $(\mathrm{z}=2.1977 \mathrm{p}=0.028)$ and anger $(\mathrm{z}=1.960, \mathrm{p}=0.05)$. The scale vigour showed a significant increase for the same period $(\mathrm{z}=2278, \mathrm{p}=0.023)$. The scale confusion showed a significant reduction for the intervals T1-T2 $(\mathrm{z}=2.701, \mathrm{p}=0.007)$ and T3-T5 $(\mathrm{z}=1.979$, $\mathrm{p}=0.048)$. Tension showed a steady reduction between T0 and T5, never reaching statistical significance though.

Perceived stress (PSS score) showed improvement in all items from T0 and T5. Nevertheless, only four items showed a statistically significant improvement: control of irritation increasing between T3 and T5 $(\mathrm{z}=2000$, $\mathrm{p}=0.048)$, Uncontrollable Anger parallel decreasing between T1 and T2 ( $\mathrm{z}=0.680, \mathrm{p}=0.046)$, Anger decreasing between $\mathrm{T} 1$ and $\mathrm{T} 4 \quad \mathrm{z}=2.212, \mathrm{p}=0.034)$ and Irritability decreasing between $\mathrm{T} 1$ and $\mathrm{T} 5$, respectively $(\mathrm{z}=2.111, \mathrm{p}=0.046)$. All changes suggest a positive effect in terms of reduction of stress. There was also a significant decrease in Self-control before the start of the intervention, between $\mathrm{T} 0$ and $\mathrm{T} 1(\mathrm{z}=2.008, \mathrm{p}=0.045)$, while it showed a trend towards improvement thereafter.

\section{Respiratory symptoms questionnaires and pulmonary function tests}

Pulmonary function tests were performed at T0, T2 and $\mathrm{T} 4$, and were analysed together with respiratory symptoms scores at the same time points. For each variable, 
Figure 2 Change in average scores of Profile Of Mood State test $(A)$ and Perceived Stress Scale (B) single items between T0 and T5.
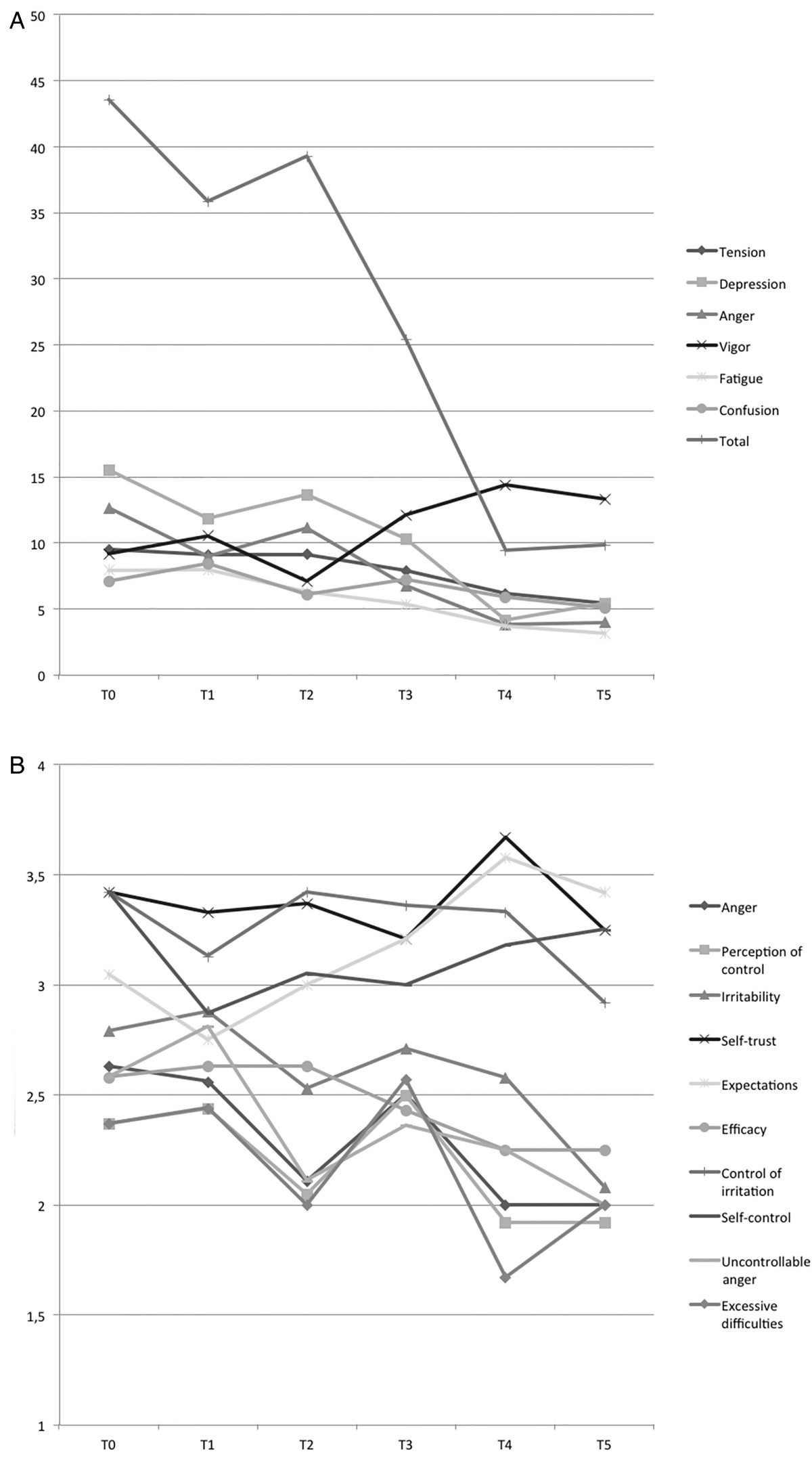

only patients for whom all three time points were available have been included.

Changes in the \% relative scores in the Shortness of Breath Questionnaire and the Cough and Sputum
Assessment Questionnaire are shown for each patient in figure 3. No statistically significant differences in questionnaire scores were observed between different time points. 
$\mathrm{SOB}$

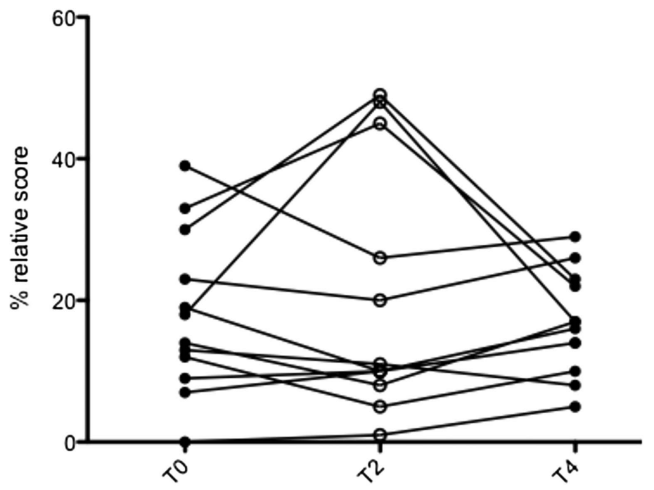

CASA-Q

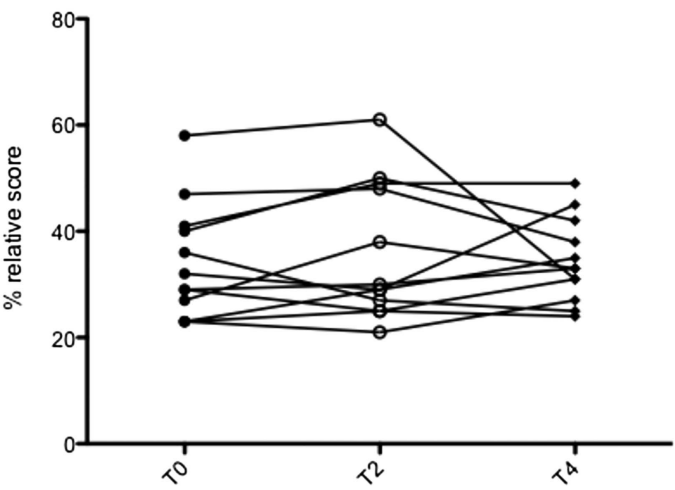

Figure 3 Change in \% relative scores of Shortness of Breath (SOB) questionnaire and Cough And Sputum Assessment Questionnaire (CASA-Q) between T0 and T4 ( $\mathrm{N}=12)$.

Similarly, changes in the serial measurements of $\%$ predicted FVC and \% predicted SB-DLCO, or in the distance walked at the 6MWT were not observed, other than those due to deteriorations associated with the progression of the underlying fibrotic lung disease (figure 4).

\section{DISCUSSION}

The findings of this 1-year prospective observational pilot study support the feasibility and safety of an MBSR program in patients affected by ILDs. Feasibility needed to be established, since one of the main practices of MBSR involves paying attention to breathing, which potentially could increase stress in these patients. The dropout rate was low $(10.5 \%)$, with two patients withdrawing from the study during the preintervention observational period, as they felt unable to attend the MBSR program regularly. All 17 patients who entered the program managed to complete the study, attending an average of eight meetings of the nine scheduled. This high completion rate is particularly pertinent as many patients had to travel (in some cases, they lived hundreds of kilometres away) and the intervention was held in winter, between January and March, and patients had to face bad weather. Although adherence to homework was not recorded (see limitations of the study below), during the 2 months program and in the follow-up period a regular daily home practice of $30-45 \mathrm{~min}$ was informally reported by $2 / 3$ of the patients; $1 / 3$ of patients admitted being less regular with home exercises.

We believe that the reasons for the good adherence to such a new approach are several. First, the setting chosen for the MBSR program was a conference room located in the Clinic for Respiratory Diseases, a familiar place for the patients. As such, the proximity to a well-known secure environment and to the familiar and trusted medical staff (although no doctors were admitted to take part in the MBSR sessions) might have worked as a facilitating factor. Second, the paucity of treatment options for many ILDs may have encouraged these greatly motivated patients to search for new approaches of care to improve their performances and quality of life. Third, feelings of loneliness and abandonment are often present in patients with rare diseases, and sharing such an experience with people affected by the same health problems, as with dedicated support groups, may be helpful. In fact, MBSR could represent a complementary strategy for developing new potential synergies and forming peer groups, as well as for helping patients acquire selfawareness and feel more empowered. Furthermore, the
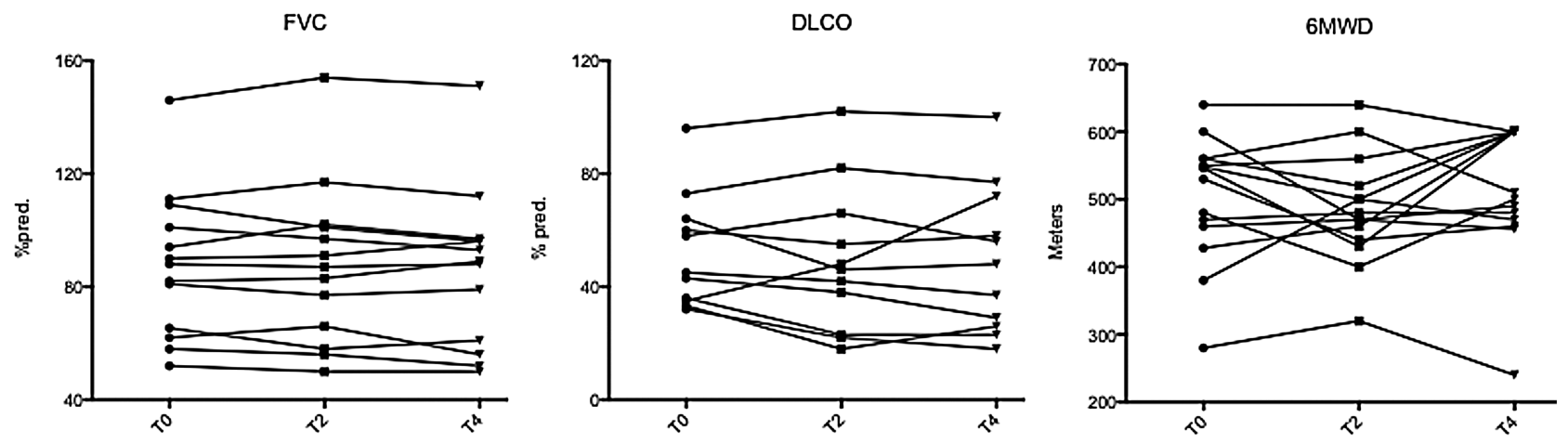

Figure 4 Change in \% predicted forced vital capacity FVC (N=13), \% predicted SB-DLCO (N=11) and 6 min walking distance $(\mathrm{N}=14)$ between T0 and T4. FVC, forced vital capacity; DLCO, diffusion lung capacity of CO; 6MWD, 6 min walking distance. 
supportive role and empathy with the mindfulness instructor may act as a non-specific factor ${ }^{13}$ improving program adherence and outcomes.

The results obtained from the questionnaires on mood and perceived stress showed a potential efficacy of the MBSR program in improving the emotional status in these patients.

The improvements in the POMS and PSS questionnaires throughout the 1-year follow-up suggests that the application of mindfulness techniques may play a role in helping patients to reduce mood disturbance and in adjusting better to their incurable disease. The mechanism of action of mindfulness is obviously different from 're-framing' in which thoughts are challenged and restructured. ${ }^{25}$ The shift of perspective, named 're-perceiving', is a more reliable mechanism. However, there might be another way how mindfulness can work, as we observed in patients an increased capacity to stay exposed to simple and preordained mental life, disrupting the strenuous process of holding on themselves and their world. This attitude of 'bearing the void' has a curious, committed approach; it is effectively sustained by deep yoga practice, that allow to listen physical sensations in a new interesting way, not confirming usual mindsets.

It is noticeable that the improvement of mood had a constant trend over time (figure 2) right after the end of the intervention period. In this period, we recorded from many patients' statements about the attractiveness of the method and their regular practice-developed in a personal blend of meditation, yoga, body scan and informal exercises. Therefore, it is possible that the follow-up periodical meetings worked as a booster for the patients' will in pursuing their home practice. It is not possible, though, to exclude some positive seasonal influence on the mood acting as a confounding factor, as the postintervention observational period covered the whole spring and summer, potentially bearing a more positive attitude.

The positive trend in the emotive sphere was not reflected by changes in respiratory symptoms of these patients, as measured by the Shortness of Breath and the CASA-Q questionnaires. Lung function and exercise tolerance were also unaffected. This discrepancy was not unexpected, however. The respiratory questionnaires focused on the ability of patients to perform daily life activities that become limited as the disease progresses, specifically dyspnoea and cough. It would be surprising if an intervention aimed at modifying the perception of a symptom had such an effect that could even alter the symptom itself and hence improve physical performances. Indeed, it has to be pointed out that there was no significant change in respiratory symptoms over the study period, which should be considered as a positive outcome in a 12-month follow-up study including patients with progressive respiratory diseases.

This pilot study has several limitations. First of all, the sample size of the study population is small. For the
POMS and the PSS tests, we performed the analysis across different time points. Although the overall change in scores between the beginning and the end of the observation period is consistent, the adoption of multiple testing carries a potential risk of generating false positives. This issue might be addressed by larger studies. ILDs are rare conditions, though, and the program itself was designed for small groups. As such, future research requires international multicentre populations. The advantage of exploring the effect of the MBSR program in people belonging to different cultural and social settings would add significant strength to the results.

Second, assessment of mood or stress is very challenging and sensible to the influence of many confounding factors: age, comorbidities (physical or psychological) and external factors (such as seasonality) can easily affect the perception of mood states and symptoms. Hence, the adoption of a properly matched control arm and randomisation would be needed to determine the real efficacy of the MBSR program in the emotional field.

Another limitation is represented by the substantial heterogeneity of the population recruited into the study in terms of disease severity and diagnosis, although the majority of patients (63\%) were affected by IPF. Again, this limitation is strictly related to the single-centre design of the study, and larger randomised and possibly multicentre studies would allow a proper stratification of patients in order to seek how this approach can impact different stages of severity in specific conditions.

Finally, in this pilot study, adherence to homework was not rigorously recorded in diaries, usually adopted for recording mindfulness practice at home. This was done in order to not apply pressure on patients with breathing difficulties. Moreover, the sample size was too small for making adequate comparisons between more and less compliant patients. Indeed, measures to monitor the adherence of participants to daily home exercises should be adopted in confirmatory studies in order to state with certainty whether pursuing mindfulness practice after the end of the program results in a consistent, long-lasting effect.

\section{CONCLUSIONS}

This pilot, single-centre, 1-year observational study demonstrates that MBSR is a feasible and safe approach in patients affected by ILDs. Our results suggest a positive effect on patients' moods, lending support for larger controlled studies with a stratified population to assess the efficacy of MBSR programs and evaluate their complementarity with existing dedicated support groups.

\section{Author affiliations}

${ }^{1}$ Southampton NIHR Respiratory Biomedical Research Unit, University Hospital Southampton, Southampton, UK

${ }^{2}$ Center for Rare Lung Disease, University Hospital Policlinico di Modena, Modena, Italy. 
${ }^{3}$ Asia Study Center, Associazione Asia, Bologna, Italy

${ }^{4}$ A.U.S.L. of Modena, Modena, Italy

${ }^{5}$ FISSPA Department, Section of Applied Psychology, Padua University, Italy

${ }^{6}$ Hospital Direction, University Hospital Policlinico di Modena, Modena, Italy

Acknowledgements RF and MPR wish to sincerely thank their meditation and yoga teacher M. Franco Bertossa of the Associazione Asia (Bologna, Italy) for his teaching and for providing inspiration and support for this work.

Contributors GS and RF have revised this version of the manuscript. Conception and design, GS, SC, RF, MPR and LR. MBSR intervention program, RF, MPR. Patients support, MO, MG. Acquisition of clinical data, GS and SC. Data analysis and interpretation, GS, SC, RF, MPR, SP, GM and KP. First drafting of manuscript GS, SC, RF. Revision of manuscript, all authors. Project supervision, LR and FL.

Competing interests None.

Ethics approval This study was granted approval from Research Ethics Committee of Modena, Italy.

Provenance and peer review Not commissioned; externally peer reviewed.

Data sharing statement No additional data are available.

Open Access This is an Open Access article distributed in accordance with the Creative Commons Attribution Non Commercial (CC BY-NC 4.0) license, which permits others to distribute, remix, adapt, build upon this work noncommercially, and license their derivative works on different terms, provided the original work is properly cited and the use is non-commercial. See: http:// creativecommons.org/licenses/by-nc/4.0/

\section{REFERENCES}

1. Raghu G, Collard HR, Egan JJ, et al. An official ATS/ERS/JRS/ ALAT statement: idiopathic pulmonary fibrosis: evidence-based guidelines for diagnosis and management. Am J Respir Crit Care Med 2011;183:788-824.

2. Ley B, Collard HR, King TE Jr. Clinical course and prediction of survival in idiopathic pulmonary fibrosis. Am J Respir Crit Care Med 2011:183:431-40.

3. Swigris JJ, Kuschner WG, Jacobs SS, et al. Health-related quality of life in patients with idiopathic pulmonary fibrosis: a systematic review. Thorax 2005;60:588-94.

4. King TE Jr, Schwarz MI, Brown K, et al. Idiopathic pulmonary fibrosis: relationship between histopathologic features and mortality. Am J Respir Crit Care Med 2001;164:1025-32.

5. Nishiyama $\mathrm{O}$, Taniguchi $\mathrm{H}$, Kondoh $\mathrm{Y}$, et al. A simple assessment of dyspnoea as a prognostic indicator in idiopathic pulmonary fibrosis. Eur Respir J 2010;36:1067-72.

6. Ryerson CJ, Collard HR, Pantilat SZ. Management of dyspnea in interstitial lung disease. Curr Opin Support Palliat Care 2010;4:69-75.
7. Bohdi B. A comprehensive manual of Abhidhamma. BPS Paryatti Editions, 2000.

8. Kabat-Zinn J. Full catastrophe living: using the wisdom of your body and mind to face stress, pain and illness. New York, NY: Dell Publishing, 1990.

9. Kabat-Zinn J. An outpatient program in behavioral medicine for chronic pain patients based on the practice of mindfulness meditation: theoretical considerations and preliminary results. Gen Hosp Psychiatry 1982;4:33-47.

10. Chambers SK, Foley E, Galt E, et al. Mindfulness groups for men with advanced prostate cancer: a pilot study to assess feasibility and effectiveness and the role of peer support. Support Care Cancer 2012;20:1183-92.

11. Shennan C, Payne S, Fenlon D. What is the evidence for the use of mindfulness-based interventions in cancer care? A review. Psychooncology 2011;20:681-97.

12. Ott MJ, Norris RL, Bauer-Wu SM. Mindfulness meditation for oncology patients: a discussion and critical review. Integr Cancer Ther 2006;5:98-108.

13. Pbert L, Madison JM, Druker S, et al. Effect of mindfulness training on asthma quality of life and lung function: a randomised controlled trial. Thorax 2012;67:769-76.

14. Fjorback LO, Arendt M, Ornbol E, et al. Mindfulness-based stress reduction and mindfulness-based cognitive therapy: a systematic review of randomized controlled trials. Acta Psychiatr Scand 2011;124:102-19.

15. Swigris JJ, Gould MK, Wilson SR. Health-related quality of life among patients with idiopathic pulmonary fibrosis. Chest 2005;127:284-94.

16. Ryerson CJ, Berkeley J, Carrieri-KohIman VL, et al. Depression and functional status are strongly associated with dyspnea in interstitial lung disease. Chest 2011;139:609-16.

17. Mcnair DM, Lorr M, Droppleman LF. Profile of Mood States Manual. Educational and Industrial Testing Services. 1992.

18. Cohen S, Kamarck T, Mermelstein R. A global measure of perceived stress. J Health Soc Behav 1983;24:385-96.

19. Eakin EG, Resnikoff PM, Prewitt LM, et al. Validation of a new dyspnea measure: the UCSD Shortness of Breath Questionnaire. University of California, San Diego. Chest 1998;113:619-24.

20. Crawford B, Monz B, Hohlfeld J, et al. Development and validation of a cough and sputum assessment questionnaire. Respir Med 2008;102:1545-55.

21. Miller MR, Hankinson J, Brusasco V, et al. Standardisation of spirometry. Eur Respir J 2005;26:319-38.

22. Wanger J, Clausen JL, Coates A, et al. Standardisation of the measurement of lung volumes. Eur Respir J 2005;26:511-22.

23. Macintyre N, Crapo RO, Viegi G, et al. Standardisation of the single-breath determination of carbon monoxide uptake in the lung Eur Respir J 2005;26:720-35.

24. Brooks D, Hunter JP, Parsons J, et al. Reliability of the two-minute walk test in individuals with transtibial amputation. Arch Phys Med Rehabil 2002;83:1562-5.

25. Greenberger D, Padesky C. Mind over mood. New York: The Guilford Press, 1995.

26. Shapiro SL, Carlson LE, Astin JA, et al. Mechanisms of mindfulness. $J$ Clin Psychol 2006;62:373-86. 